\title{
A Generalized Reverse Jacket Transform
}

\author{
Moon Ho Lee, Senior Member, IEEE, B. Sundar Rajan, Senior Member, IEEE, and J. Y. Park, Member, IEEE
}

\begin{abstract}
Generalization of the well-known Walsh-Hadamard transform (WHT), namely center-weighted Hadamard transform (CWHT) and complex reverse-jacket transform (CRJT) have been proposed and their fast implementation and simple index generation algorithms have recently been reported. These transforms are of size $2^{r} \times 2^{r}$ for integral values or $r$, and defined in terms of binary radix representation of integers. In this paper, using appropriate mixed-radix representation of integers, we present a generalized transform called general reverse jacket transform (GRJT) that unifies all the three classes of transforms, WHT, CWHT, and CRJT, and also applicable for any even length vectors, that is of size $2 r \times 2 r$. A subclass of GRJT which includes CRJT (but not CWHT) is applicable for finite fields and useful for constructing error control codes.
\end{abstract}

Index Terms-Error-control coding, image-coding, reverse-jacket transform, Walsh-Hadamard transform.

\section{INTRODUCTION}

$\mathbf{T}$ HE WALSH-HADAMARD transform (WHT) and discrete Fourier transform (DFT) are used widely in signal processing [1], [3], in particular image coding and processing [2] and error-control coding [13], [4], [10]. Recently, variations of these two transforms called center weighted Hadamard transform (CWHT) and complex reverse jacket transform (CRJT) [5]-[7], [9] have been reported and their applications in image processing and communications have been pointed out [15].

Both the CWHT and the CRJT include the WHT as a special case. The CRJT uses the complex number $j=\sqrt{-1}$ which is a fourth root of unity. In this paper, we first generalize the CRJT by extending it to any $2 n$th root of unity, for any integer value of $n$. This extended CRJT is well defined for any finite field apart from the complex field and is useful in constructing new classes of error correcting codes. Then we obtain a further generalized transform called generalized reverse jacket transform (GRJT) that includes the CWHT as well as the extended CRJT and hence the WHT as special cases. Fig. 1 illustrates the interrelationship among these transforms.

CRJT has been used to construct optimal bipolar sequence [14] and it is hoped that the extended CRJT presented in this paper will lead to good multilevel sequences. Moreover, WHT is used in practical design of substitution boxes (S-box) in data

Manuscript received June 22, 2000; revised July 24, 2001. This paper was recommended by Associate Editor B. Baykal.

M. H. Lee is with the Institute of Information and Communication, Chonbuk National University, Chonju 560-756, Korea (e-mail: moonho@ chonbuk.ac.kr).

B. S. Rajan is with the Department of Communication Engineering, Indian Institute of Science, Bangalore, 560012 India (e-mail: bsrajan@ece.iisc.ernet.in).

J. Y. Park is with the Department of Electrical and Electronics Engineering, Seonam University, Namwon City, Chonbuk-590 711, Korea (e-mail: jypark@tiger.seonam.ac.kr).

Publisher Item Identifier S 1057-7130(01)09128-5. encryption standard (DES) [16] and the generalized transforms presented here may be used in this design.

In this introductory section, we briefly discuss the WHT, CWHT, and CRJT, mainly to give their definitions and fix the terminology and notations.

\section{A. The WHT}

For a $n=2^{r}$ length real vector $\left(a_{\circ}, a_{1}, \ldots, a_{n-1}\right)$ the transform vector is a $n$-length real vector $\left(A_{0}, A_{1}, \ldots, A_{n-1}\right)$ given by

$$
A_{j}=\sum_{i=0}^{n-1}(-1)^{\langle j, i\rangle} a_{i}, \quad j=0,1, \ldots, n-1
$$

where the modulo- 2 inner product $\langle j, i\rangle$ is given by

$$
\langle j, i\rangle=j_{r-1} i_{r-1} \oplus j_{r-2} i_{r-2} \oplus \cdots \oplus j_{0} i_{0}
$$

where $\oplus$ denotes modulo two addition, in terms of radix-2 representation of integers $i \in\{0,1, \ldots, n-1\}$, denoted by

$$
\langle i\rangle=\left\langle i_{r-1}, i_{r-2}, \ldots, i_{0}\right\rangle
$$

where

$$
i=i_{r-1} 2^{r-1}+i_{r-2} 2^{r-2}+\cdots+i_{1} 2+i_{0}
$$

and $i_{\lambda}=0,1$ for $\lambda=0,1, \ldots, r-1$. The inverse transform is given by

$$
a_{j}=\frac{1}{n} \sum_{i=0}^{n-1}(-1)^{\langle j, i\rangle} A_{i}, \quad j=0,1, \ldots, n-1 .
$$

\section{B. The CWHT}

The CWHT is obtained by weighting the center portion of the transform matrix given by (1) and is given by

$$
\begin{gathered}
A_{j}=\sum_{i=0}^{n-1}(-1)^{\langle j, i\rangle}(w)^{\left(i_{r-1} \ominus i_{r-2}\right)\left(j_{r-1} \Theta j_{r-2}\right)} a_{i}, \\
j=0,1, \ldots, n-1
\end{gathered}
$$

where $w$ is any real number is the weight. The inverse transform is given by

$$
\begin{gathered}
a_{j}=\frac{1}{n} \sum_{i=0}^{n-1}(-1)^{\langle j, i\rangle}\left(w^{-1}\right)^{\left(i_{r-1} \Theta i_{r-2}\right)\left(j_{r-1} \Theta j_{r-2}\right)} A_{i}, \\
j=0,1, \ldots, n-1 .
\end{gathered}
$$




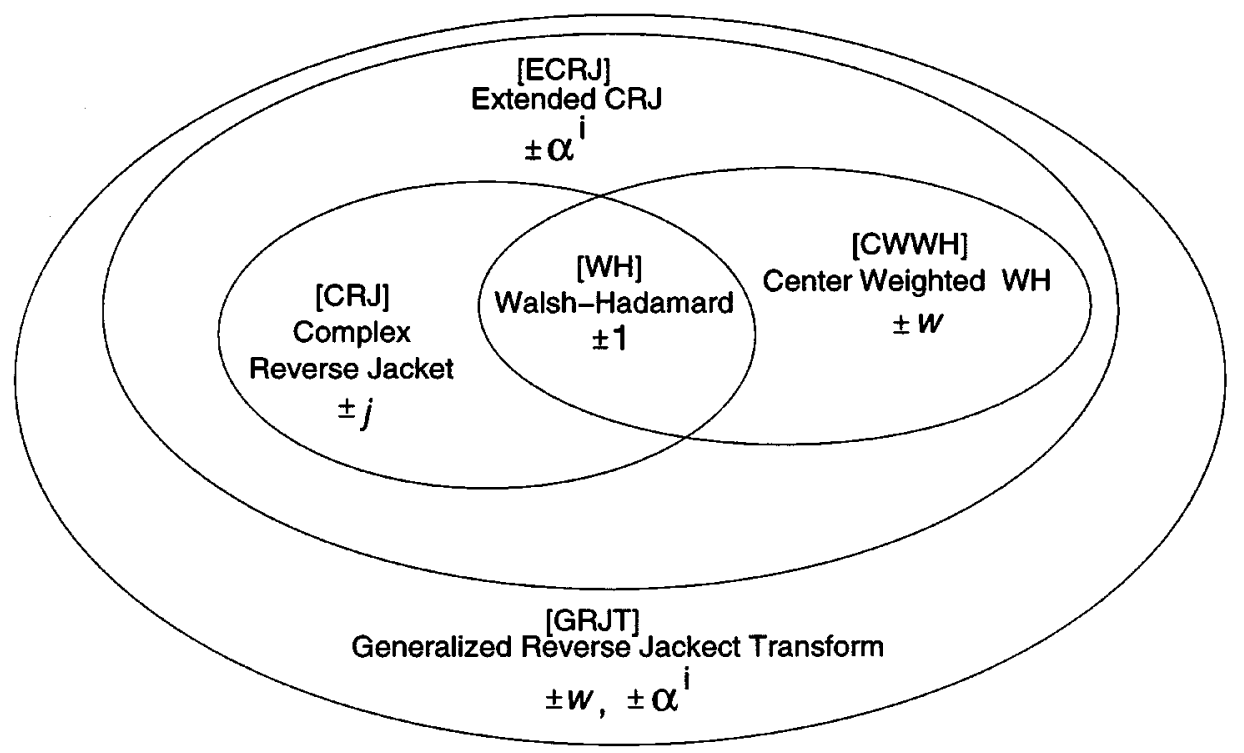

Fig. 1. Interrelationship among the transforms.

Notice that for $w=1$ the CWHT is same as the WHT. The $4 \times 4$ and $8 \times 8$ CWHT matrices corresponding to the weight $w$ are, respectively

$$
[W]_{4 \times 4}=\left[\begin{array}{rrrr}
1 & 1 & 1 & 1 \\
1 & -w & w & -1 \\
1 & w & -w & -1 \\
1 & -1 & -1 & 1
\end{array}\right]
$$

and

$$
[W]_{8 \times 8}=\left[\begin{array}{rrrrrrrr}
1 & 1 & 1 & 1 & 1 & 1 & 1 & 1 \\
1 & -1 & 1 & -1 & 1 & -1 & 1 & -1 \\
1 & 1 & -w & -w & w & w & -1 & -1 \\
1 & -1 & -w & w & w & -w & -1 & 1 \\
1 & 1 & w & w & -w & -w & -1 & -1 \\
1 & -1 & w & -w & -w & w & -1 & 1 \\
1 & 1 & -1 & -1 & -1 & -1 & 1 & 1 \\
1 & -1 & -1 & 1 & -1 & 1 & 1 & -1
\end{array}\right]
$$

and the inverses, respectively, are

$$
\begin{aligned}
& {[W]_{4 \times 4}^{-1}} \\
& \quad=\frac{1}{4}\left[\begin{array}{rrrr}
1 & 1 & 1 & 1 \\
1 & -w^{-1} & w^{-1} & -1 \\
1 & w^{-1} & -w^{-1} & -1 \\
1 & -1 & -1 & 1
\end{array}\right]
\end{aligned}
$$

and

$$
\begin{aligned}
& {[W]_{8 \times 8}^{-1}} \\
& \quad=\frac{1}{8}\left[\begin{array}{rrrrrrrr}
1 & 1 & 1 & 1 & 1 & 1 & 1 & 1 \\
1 & -1 & 1 & -1 & 1 & -1 & 1 & -1 \\
1 & 1 & -w^{-1} & -w^{-1} & w^{-1} & w^{-1} & -1 & -1 \\
1 & -1 & -w^{-1} & w^{-1} & w^{-1} & -w^{-1} & -1 & 1 \\
1 & 1 & w^{-1} & w^{-1} & -w^{-1} & -w^{-1} & -1 & -1 \\
1 & -1 & w^{-1} & -w^{-1} & -w^{-1} & w^{-1} & -1 & -1 \\
1 & 1 & -1 & -1 & -1 & -1 & 1 & 1 \\
1 & -1 & -1 & 1 & -1 & 1 & 1 & -1
\end{array}\right] .
\end{aligned}
$$

\section{The CRJT}

In [6], a class of transform called CRJT is constructed by replacing the weight $w$ in (2) by the complex number $j=\sqrt{-1}$, and this CRJT is orthogonal and a fast algorithm to compute CRJT is also given. In [8] a simple binary index generation algorithm is discussed for the CRJT and its applications in coded modulation pointed out. However, it is not discussed whether one can use other complex numbers, instead of $-j$ or $j$.

The $4 \times 4$ and the $8 \times 8$ CRJT are shown below as

$$
[C]_{4 \times 4}=\left[\begin{array}{rrrr}
1 & 1 & 1 & 1 \\
1 & -j & j & -1 \\
1 & j & -j & -1 \\
1 & -1 & -1 & 1
\end{array}\right]
$$

and

$$
[C]_{8 \times 8}=\left[\begin{array}{rrrrrrrr}
1 & 1 & 1 & 1 & 1 & 1 & 1 & 1 \\
1 & -1 & 1 & -1 & 1 & -1 & 1 & -1 \\
1 & 1 & -j & -j & j & j & -1 & -1 \\
1 & -1 & -j & j & j & -j & -1 & 1 \\
1 & 1 & j & j & -j & -j & -1 & -1 \\
1 & -1 & j & -j & -j & j & -1 & 1 \\
1 & 1 & -1 & -1 & -1 & -1 & 1 & 1 \\
1 & -1 & -1 & 1 & -1 & 1 & 1 & -1
\end{array}\right] .
$$

Observe that $-j$ is a primitive fourth root of unity on the complex unit circle and we show that by replacing $j$ with any $2 n$th (for any positive integer $n$ ) complex root of unity and using appropriate mixed-radix indexing scheme CRJT can be extended to any even length vectors. This extended CRJT is orthogonal.

The content of the paper is organized as follows: In the next section, we extend the $4 \times 4$ CRJT given by (7) to any $2 n \times 2 n$ by using a $2 n$th complex root of unity instead of $j=\sqrt{-1}$ as an intermediate step toward a GRJT. By showing the connection with DFT, this extended CRJT transform is shown to be orthogonal. It is also observed that the extended CRJT is applicable for appropriate finite field instead of complex field and new class of error correcting codes can be obtained. In Section III, we unify the CWHT and the extended CRJT to obtain the GRJT, which is 
not necessarily orthogonal. Section IV contains few concluding remarks and several directions for further research.

\section{THE EXTENDED CRJT}

All the transforms discussed in the previous section, WHT, CWHT, and CRJT are defined for lengths that are powers of two only, or equivalently the corresponding transform matrices are of type $2^{r} \times 2^{r}$ for some positive integer $r$. This is due to the fact that the CWHT is nothing but the center portion of the matrix weighted by $w$ and in CRJT it is due to the fact that $j=\sqrt{-1}$ is an element of order 4. If, instead of $j$ which is a fourth root of unity, $\alpha$ a $2 n$th root of unity is used then instead of a $4 \times 4$ matrix, a $2 n \times 2 n$ matrix results. In this section we obtain this matrix, using a mixed radix representation of integers between zero and $2 n-1$. A class of optimal bipolar sequences has been obtained in [14] using CRJT and it is hoped that the generalization presented in this section will lead to new classes of optimal sequences.

Definition 1: Let $n \geq 2$ be an arbitrary positive integer and $\alpha$ denote a primitive $2 n$th root of unity on the complex circle. For any real or complex $2 n$-length vector $\left(a_{0}, a_{1}, \ldots, a_{2 n-1}\right)$ the GRJT vector $\left(A_{0}, A_{1}, \ldots, A_{2 n-1}\right)$ is given by

$$
\begin{aligned}
& A_{j}= \\
& \sum_{i=0}^{2 n-1} \alpha^{\left[n j_{1}+\left(1-j_{1}\right) j_{0}+j_{1}\left(n-1-j_{0}\right)\right]\left[n i_{1}+\left(1-i_{1}\right) i_{0}+i_{1}\left(n-1-i_{0}\right)\right]} a_{i}
\end{aligned}
$$

where, for any integer $i \in\{0,1, \ldots, 2 n-1\}$ the mixed-radix representation of it is given by $\langle i\rangle=\left\langle i_{1}, i_{0}\right\rangle$ where $i=n i_{1}+i_{0}$ and $i_{1} \in\{0,1\}$ and $i_{0} \in\{0,1, \ldots, n-1\}$.

It is easy to check that the inverse GRJT is given by

$$
\begin{aligned}
a_{j}= & \frac{1}{2 n} \sum_{i=0}^{2 n-1} \\
& \cdot \alpha^{-\left[n j_{1}+\left(1-j_{1}\right) j_{0}+j_{1}\left(n-1-j_{0}\right)\right]\left[n i_{1}+\left(1-i_{1}\right) i_{0}+i_{1}\left(n-1-i_{0}\right)\right]} A_{i} .
\end{aligned}
$$

Observe that when $n=2$, Definition 1 gives the $4 \times 4$ CRJT given by (7).

Example 1: Let $n=3$. Then $\alpha=e^{\sqrt{-1}(\pi / 3)}$. The transform matrix and inverse matrix corresponding to Definition 1 are

$$
[R]_{6 \times 6}=\left[\begin{array}{rrrrrr}
1 & 1 & 1 & 1 & 1 & 1 \\
1 & \alpha & \alpha^{2} & \alpha^{5} & \alpha^{4} & -1 \\
1 & \alpha^{2} & \alpha^{4} & \alpha^{4} & \alpha^{2} & 1 \\
1 & \alpha^{5} & \alpha^{4} & \alpha & \alpha^{2} & -1 \\
1 & \alpha^{4} & \alpha^{2} & \alpha^{2} & \alpha^{4} & 1 \\
1 & -1 & 1 & -1 & 1 & -1
\end{array}\right]
$$

and

$$
[R]_{6 \times 6}^{-1}=\frac{1}{6}\left[\begin{array}{rrrrrr}
1 & 1 & 1 & 1 & 1 & 1 \\
1 & \alpha^{5} & \alpha^{4} & \alpha & \alpha^{2} & -1 \\
1 & \alpha^{4} & \alpha^{2} & \alpha^{2} & \alpha^{4} & 1 \\
1 & \alpha & \alpha^{2} & \alpha^{5} & \alpha^{4} & -1 \\
1 & \alpha^{2} & \alpha^{4} & \alpha^{4} & \alpha^{2} & 1 \\
1 & -1 & 1 & -1 & 1 & -1
\end{array}\right]
$$

The following theorem shows that the transforms given by Definition 1 is orthogonal for all values of $n$.
Theorem 1: The transform given by Definition 1 is unitary.

Proof: The Theorem follows from the fact that it is a permuted version of the $2 n$ length DFT, given by

$$
A_{j}=\sum_{i=0}^{2 n-1} \alpha^{i j} a_{i} ; \quad j=0,1, \ldots, 2 n-1
$$

which is unitary. To be precise, starting from the DFT matrix, the permutation

$$
j \Rightarrow n j_{1}+\left(1-j_{1}\right) j_{0}+j_{1}\left(n-1-j_{0}\right)
$$

retains the first $n$ columns unchanged and reverses the last $n$ columns. After this column permutation, the permutation

$$
i \Rightarrow n i_{1}+\left(1-i_{1}\right) i_{0}+i_{1}\left(n-1-i_{0}\right)
$$

retains the first $n$ rows unchanged and reverses the last $n$ rows. Since column permutation and row permutation does not affect the value of inner products of columns and rows, unitariness is unaffected.

Notice that the matrix shown in (8) is not obtainable from the transform of Definition 1. This calls for the further generalization of Definition 1. Definition 2 gives such a transform which we call extended CRJT. For this definition, we need the following definition of mixed-radix representation of integers from $\{0,1, \ldots, N-1\}$ for $N$ of the form $N=2^{l} n$, where $l$ and $n$ are positive integers.

Definition 2: Let $N=2^{l} n$, where $n$ and $l$ are arbitrary positive integers. An integer $i \in\{0,1, \ldots, N-1\}$ has a representation of the form

$$
i=i_{l}\left(n 2^{l-1}\right)+i_{l-1} 2^{l-1}+i_{l-2} 2^{l-2}+\cdots+i_{1} 2+i_{0}
$$

where $i_{l}$ and $i_{\lambda}$ for $\lambda=l-2, l-3, \ldots, 1,0$ take values from $\{0,1\}$ and $i_{l-1} \in\{0,1, \ldots, n-1\}$, and this will be represented by

$$
i=\left\langle i_{l}, i_{l-1}, i_{l-2}, \ldots, i_{1}, i_{0}\right\rangle .
$$

Observe that for the same value of $N$ if $l$ and $n$ are different, the corresponding mixed-radix number system is different. The following example exemplifies this.

Example 2: For $N=2^{2} 3, N=2^{3} 3$ and $N=2^{2} 6$, the mixed-radix representation of integers from $\{0,1, \ldots, N-1\}$ is shown in Table I.

Definition 3 (Extended CRJT): Let $N=2^{l} n$, where $l$ and $n$ are positive integers. For any real or complex valued $N$-length vector $\left(a_{0}, a_{1}, \ldots, a_{N-1}\right)$ the extended CRJT vector is the $N$-length complex vector $\left(A_{0}, A_{1}, \ldots, A_{N-1}\right)$ given by (12), shown at the bottom of the next page, and where $\alpha$ is a primitive $n$th root of unity on the complex unit circle and

$$
\ll j, i \gg=j_{l-2} i_{l-2} \oplus j_{l-3} i_{l-3} \oplus \cdots \oplus j_{1} i_{1} \oplus j_{0} i_{0}
$$

where $j$ and $i$ are represented by the mixed-radix representation of Definition 2.

Notice that the definition of $\langle j, i \gg$ is modulo 2 inner product of $j$ and $i$ without the components $i_{l}, j_{l}, i_{l-1}$ and $j_{l-1}$.

It is easy to verify that the inverse of the extended CRJT is given in (12a) as shown at the bottom of the next page. 
TABLE I

MIXED-RADIX REPRESENTATION CORRESPONDING TO EXAMPLE 2

\begin{tabular}{|c|c|c|c|c|c|c|c|c|c|}
\hline \multicolumn{2}{|c|}{$N=2^{2} 3$} & \multicolumn{4}{|c|}{$N=2^{3} 3$} & \multicolumn{4}{|c|}{$N=2^{2} 6$} \\
\hline $\bar{i}$ & $i_{2} i_{1} i_{0}$ & $i$ & $i_{3} i_{2} i_{1} i_{0}$ & $i$ & $i_{3} i_{2} i_{1} i_{0}$ & $i$ & $\overline{i_{2} i_{1} i_{0}}$ & $i$ & $i_{2} i_{1} i_{0}$ \\
\hline 0 & 000 & 0 & 0000 & 12 & 1000 & 0 & 000 & 12 & 100 \\
\hline 1 & 001 & 1 & 0001 & 13 & 1001 & 1 & 001 & 13 & 101 \\
\hline 2 & 010 & 2 & 0010 & 14 & 1010 & 2 & 010 & 14 & 110 \\
\hline 3 & 011 & 3 & 0011 & 15 & 1011 & 3 & 011 & 15 & 111 \\
\hline 4 & 020 & 4 & 0100 & 16 & 1100 & 4 & 020 & 16 & 120 \\
\hline 5 & 021 & 5 & 0101 & 17 & 1101 & 5 & 021 & 17 & 121 \\
\hline 6 & 100 & 6 & 0110 & 18 & 1110 & 6 & 030 & 18 & 130 \\
\hline 7 & 101 & 7 & 0111 & 19 & 1111 & 7 & 031 & 19 & 131 \\
\hline 8 & 110 & 8 & 0200 & 20 & 1200 & 8 & 040 & 20 & 140 \\
\hline 9 & 111 & 9 & 0201 & 21 & 1201 & 9 & 041 & 21 & 141 \\
\hline 10 & 120 & 10 & 0210 & 22 & 1210 & 10 & 050 & 22 & 150 \\
\hline 11 & 121 & 11 & 0211 & 23 & 1211 & 11 & 051 & 23 & 151 \\
\hline
\end{tabular}

Now, the matrix shown in (8) is obtained from the Definition 3 , for $l=2$ and $n=2$ by taking $\alpha=j$, which is a fourth root of unity in the complex circle. Moreover, Definition 3 gives transforms for lengths that are not powers of 2 as shown by the following example.

Example 3: For $N=12=2^{2} 3$, the extended CRJT is same as the matrix shown in (21) with $\omega$ removed from wherever it appears or equivalently with $\omega=1$.

Now we formally show that the transform given by Definition 3 is orthogonal.

Theorem 2: The extended CRJT is unitary.

Proof: Let $\mathrm{H}_{2}$ denote the $2 \times 2$ Walsh-Hadamard matrix

$$
\left[\begin{array}{rr}
1 & 1 \\
1 & -1
\end{array}\right]
$$

$\mathbf{C}_{2 n}$ the transform matrix given by Definition 1 and $\mathcal{C}_{l, n}$ denote the matrix given by Definition 3. Also, let $A^{* t}$ denote the conjugate transposed matrix of a matrix $A$ with complex numbers. Then we have

$$
\mathcal{C}_{l, n}=H_{2} \otimes H_{2} \otimes \cdots(l-1) \times \cdots \otimes H_{2} \otimes \mathbf{C}_{2 n}
$$

where $\otimes$ denotes the Kronecker product. (For properties of Kronecker products, see [17]). The Kronecker product has the following property:

$$
(A \otimes B)(C \otimes D)=(A C) \otimes(B D)
$$

where $A, B, C$ and $D$ are matrices. Using the above property, we have

$$
\begin{aligned}
\left(\mathcal{C}_{l, n}\right)\left(\mathcal{C}_{l, n}^{* t}\right)= & {\left[H_{2} \otimes \cdots(l-1) \times \cdots \otimes H_{2} \otimes \mathbf{C}_{2 n}\right] } \\
& \cdot\left[H_{2} \otimes \cdots(l-1) \times \cdots \otimes H_{2} \otimes \mathbf{C}_{2 n}^{* t}\right] \\
= & I_{2} \otimes \cdots(l-1) \times \cdots I_{2} \otimes I_{2 n} \\
= & I_{2^{l} n}
\end{aligned}
$$

where $I_{m}$ denotes the $m \times m$ identity matrix. This completes the proof.

Unitary transforms are useful in image enhancement using filtering in the transform domain. For a detailed discussion of transform operations and generalized linear filtering using the unitary transforms discrete cosine transform (DCT), WHT, and DFT, the readers are referred to [18]. Since extended CRJT is unitary, this can be used in such transform processing. In the following subsection we discuss an application of extended CRJT in the area of error control coding.

\section{A. The Extended CRJT Over Finite Fields}

In this section, we discuss the applicability of CRJT over finite fields and its usefulness to construct codes. For basic properties of finite fields and techniques of construction the reader is referred to [13]. Our purpose/intention is to demonstrate the applicability of CRJT over finite fields in constructing codes not actually giving all the details of the code construction.

$$
A_{j}=\sum_{i=0}^{N-1}(-1)^{\ll j, i \gg} \alpha^{\left[n j_{l}+\left(1-j_{l}\right) j_{l-1}+j_{l}\left(n-1-j_{l-1}\right)\right]\left[n i_{l}+\left(1-i_{l}\right) j_{l-1}+i_{l}\left(n-1-i_{l-1}\right)\right]} a_{i}, \quad j=0,1, \ldots, N-1
$$

$$
a_{j}=\frac{1}{N} \sum_{i=0}^{N-1}(-1)^{\ll j, i \gg} \alpha^{-\left[n j_{1}+\left(1-j_{l}\right) j_{l-1}+j_{l}\left(n-1-j_{l-1}\right)\right]\left[n i_{1}+\left(1-i_{l}\right) i_{l-1}+i_{l}\left(n-1-i_{l-1}\right)\right]} A_{i}, \quad j=0,1, \ldots, N-1
$$


The use of DFT over finite fields to describe cyclic codes and for decoding is well known [13], [4]. Recently, WHT over finite fields have been used to describe dyadic codes and quasicyclic dyadic codes (see [10] and the references therein). The DFT is an orthogonal transform that maps cyclic convolution to pointwise multiplication-this is the property used to describe cyclic codes over finite fields. Since the extended CRJT is also orthogonal it can be used to obtain codes in the same way as DFT is used.

We first define the extended CRJT as applicable for finite fields as follows:

Definition 4: Let $N=2^{l} n$, where $l, n$ are as in Definition 3 along with the mixed-radix integer representation. Also let $F_{q}$ be a finite field with $q$ elements where $N$ and $q$ are relatively prime and $F_{q^{m}}$ be an extension field of $F_{q}$ where $m$ is the least integer such that $2 n$ divides $q^{m}-1$ and $2 n h=q^{m}-1$. Let $\alpha$ be a primitive element of $F_{q^{m}}$. Then the element $\alpha^{\left(q^{m}-1\right) / h}$, which we denote by $\beta$ has order $2 n$. Then the extended CRJT equation (12) is modified as shown in (12b) at the bottom of the page, where $\left(a_{0}, a_{1}, \ldots, a_{N-1}\right)$ is a vector over $F_{q}$ and $\left(A_{0}, A_{1}, \ldots, A_{N-1}\right)$ is its transform vector over $F_{q^{m}}$.

We construct two examples of transform matrices shown here.

Example 4: Let $N=6, l=1, n=3$ and $q=5$. Then $m=2, h=4$ and $F_{25}$ constructed with the prime polynomial $x^{2}+x+1$ is the set

\section{$00,10,20,30,40,01,11,21,31,41,02,12,22,32,42$, \\ $03,13,23,33,43,04,14,24,34,44$}

where $a b$ denotes $a+b x$ where $a, b \in 0,1,2,3,4$. Multiplication of two elements is carried out modulo $x^{2}+x+1$. The element 13 is primitive and we take $\alpha=13$. Then $\beta=\alpha^{4}=04$. For convenience, we show at the bottom of the page, all the nonzero elements in terms of the power of $\alpha$. The extended CRJT in this case is

$$
[F]_{6 \times 6}=\left[\begin{array}{cccccc}
10 & 10 & 10 & 10 & 10 & 10 \\
10 & 04 & 44 & 11 & 01 & 40 \\
10 & 44 & 01 & 01 & 44 & 10 \\
10 & 11 & 01 & 04 & 44 & 40 \\
10 & 01 & 44 & 44 & 01 & 10 \\
10 & 40 & 10 & 40 & 10 & 40
\end{array}\right]
$$

Example 5: In Example 4, if $l$ is changed to 2, then $N=12$ and all other values remain same and the corresponding extended CRJT is shown

$$
\begin{aligned}
& {[F]_{12 \times 12}} \\
& =\left[\begin{array}{llllllllllll}
10 & 10 & 10 & 10 & 10 & 10 & 10 & 10 & 10 & 10 & 10 & 10 \\
10 & 40 & 10 & 40 & 10 & 40 & 10 & 40 & 10 & 40 & 10 & 40 \\
10 & 10 & 04 & 04 & 44 & 44 & 11 & 11 & 01 & 01 & 40 & 40 \\
10 & 40 & 04 & 01 & 44 & 11 & 11 & 44 & 01 & 04 & 40 & 10 \\
10 & 10 & 44 & 44 & 01 & 01 & 01 & 01 & 44 & 44 & 10 & 10 \\
10 & 40 & 44 & 11 & 01 & 04 & 01 & 04 & 44 & 11 & 10 & 40 \\
10 & 10 & 11 & 11 & 01 & 01 & 01 & 01 & 44 & 44 & 40 & 40 \\
10 & 40 & 11 & 44 & 01 & 04 & 01 & 04 & 44 & 11 & 40 & 10 \\
10 & 10 & 01 & 01 & 44 & 44 & 44 & 44 & 01 & 01 & 10 & 10 \\
10 & 40 & 01 & 04 & 44 & 11 & 44 & 11 & 01 & 04 & 10 & 40 \\
10 & 10 & 40 & 40 & 10 & 10 & 40 & 40 & 10 & 10 & 40 & 40 \\
10 & 40 & 40 & 10 & 10 & 40 & 40 & 10 & 10 & 40 & 40 & 10
\end{array}\right] .
\end{aligned}
$$

\section{THE GRJT}

In this section, we unify the CWHT and the extended CRJT obtained in the previous section. When the CWHT is used on a $2^{r} \times 2^{r}$ size image, pixels in the first and the last $2^{r-2}$ rows are not weighted and among the rest $2^{r-1}$ rows, pixels in the first and the last $2^{r-2}$ columns are not weighted. The ratio of the number of pixels weighted to the total number of pixels is one-quarter, and this ratio is independent of the length of the transform. This ratio in the following GRJT is not fixed and depends on the length of the transform as we shall see in the sequel.

Definition 5 (GRJT): Let $N=2^{l} n$, where $l$ and $n$ are positive integers and $\omega$ any real number. For any real or complex valued $N$-length vector $\left(a_{0}, a_{1}, \ldots, a_{N-1}\right)$ the GRJT vector is the $N$-length complex vector $\left(A_{0}, A_{1}, \ldots, A_{N-1}\right)$ given by (19) at the top of the next page, where $\alpha$ is a primitive $2 n$th root of unity on the complex unit circle

$$
\ll j, i \gg=j_{l-2} i_{l-2} \oplus j_{l-3} i_{l-3} \oplus \cdots \oplus j_{1} i_{1} \oplus j_{0} i_{0}
$$

and $\Delta(i)=0$ if $i_{l}+i_{l-1}(\operatorname{modulo} n)=0$ and $\Delta(i)=1$ otherwise.

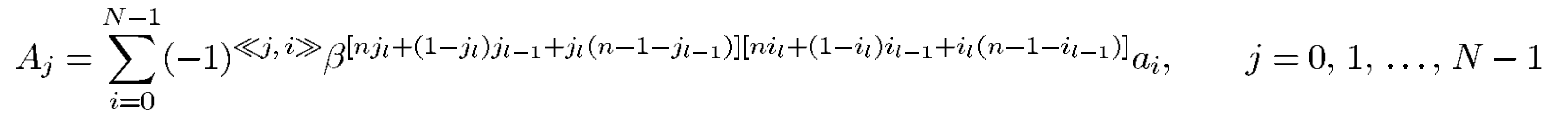

\begin{tabular}{|c|c|c|c|c|c|c|c|c|c|c|c|c|c|c|}
\hline$\alpha^{0}$ & $\alpha^{1}$ & $\alpha^{2}$ & $\alpha^{3}$ & $\alpha^{4}$ & $\alpha^{5}$ & $\alpha^{6}$ & $\alpha^{7}$ & $\alpha^{8}$ & $\alpha^{9}$ & $\alpha^{10}$ & $\alpha^{11}$ & $\alpha^{12}$ & $\alpha^{13}$ & $\alpha^{14}$ \\
$\alpha^{15}$ & $\alpha^{16}$ & $\alpha^{17}$ & $\alpha^{18}$ & $\alpha^{19}$ & $\alpha^{20}$ & $\alpha^{21}$ & $\alpha^{22}$ & $\alpha^{23}$ & \multicolumn{7}{|c|}{$\mid$} \\
\hline 10 & 13 & 22 & 12 & 04 & 32 & 20 & 21 & 44 & 24 & 03 & 14 & 40 & 42 & 33 \\
43 & 01 & 23 & 30 & 34 & 11 & 31 & 02 & 41 & & & & & & \\
\hline
\end{tabular}




$$
A_{j}=\sum_{i=0}^{N-1}(-1)^{\ll j, i \gg} \omega^{\Delta(i) \Delta(j)} \alpha^{\left[n j_{l}+\left(1-j_{l}\right) j_{l-1}+j_{l}\left(n-1-j_{l-1}\right)\right]\left[n i_{l}+\left(1-i_{l}\right) i_{l-1}+i_{l}\left(n-1-j_{l-1}\right)\right]} a_{i}
$$

$$
a_{j}=\frac{1}{N} \sum_{i=0}^{N-1}(-1)^{\ll j, i \gg}\left(\omega^{-1}\right)^{\Delta(i) \Delta(j)} \alpha^{-\left[n j_{l}+\left(1-j_{l}\right) j_{l-1}+j_{l}\left(n-1-j_{l-1}\right)\right]\left[n i_{l}+\left(1-i_{l}\right) i_{l-1}+i_{1}\left(n-1-i_{l-1}\right)\right]} A_{i}
$$

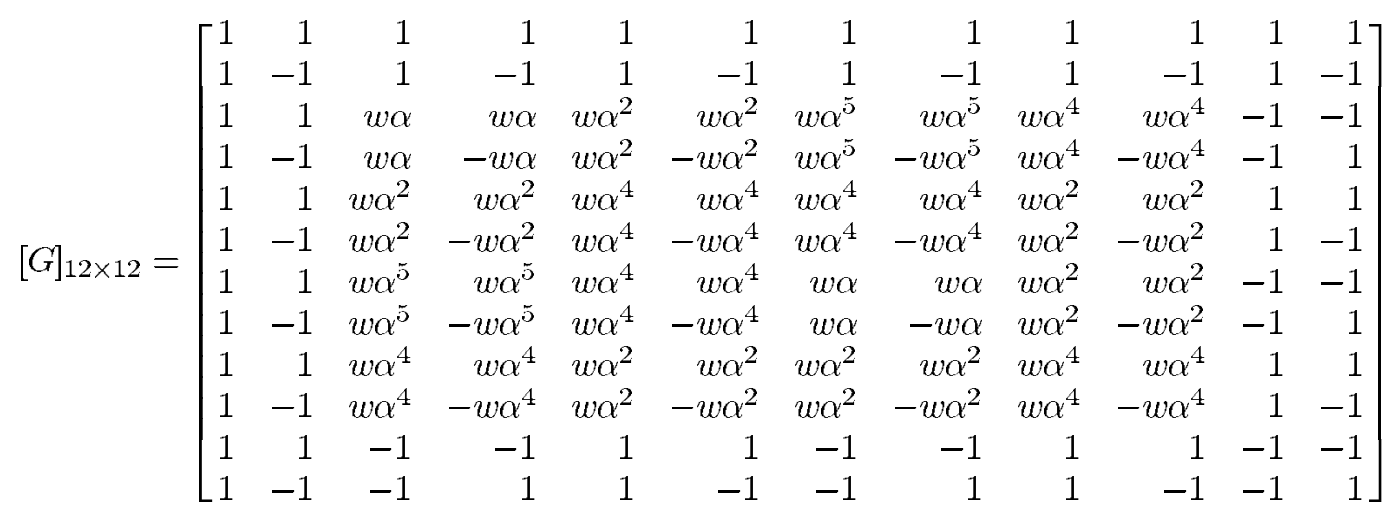

It is easy to check that the inverse transform is given by (20) shown at the top of the page.

Example 6: The GRJT matrix for $l=2$ and $n=3$ is shown in (21), at the bottom of the page, where $\omega$ is the weight and $\alpha=e^{\sqrt{-1}(\pi / 3)}$.

The WHT, CWHT, CRJT, and the extended CRJT are all special cases of the GRJT, obtainable from Definition 4, as follows:

- $\alpha=\omega=1$ gives the WHT;

- $\alpha=1$ gives the CWHT;

- $\omega=1$ and $\alpha=-j$ gives the CRJT;

- $\omega=1$ gives the entended CRJT.

Now, it is straight forward to see that in the GRJT the ratio of the number of pixels that are weighted to the total number of pixels is

$$
\frac{2 l(n-1)}{2^{l} n}
$$

and this ratio depends on $l$ and $n$. For instance, for 24 length transform $l=2, n=6$ leads to the ratio $5 / 6$ whereas $l=$ $3, n=3$ leads to the ratio $1 / 2$. Recall that for the CRJT this ratio is fixed and always one-quarter.

\section{CONCLUSION}

In this paper we have extended the CRJT which is defined using the so called imaginary number $j$ to the extended CRJT that uses a complex $2 n$th root of unity where $n$ is any positive integer greater than or equal to 2. Further, the WHT, CWHT, CRJT, and extended CRJT have all been shown to be obtainable as special cases of the GRJT. The application of these transforms to image processing, error control coding and sequences have been cited. The possible directions for further research are as follows:

- Optimal bipolar sequences have been obtained using the CRJT and naturally the extended CRJT correspond to mul- tiple valued sequences and constitute an interesting area to investigate further.

- Extension of the fast algorithm and the index generation algorithm given in [6] and [8] for CRJT to the GRJT is not straight forward because of the mixed-radix representation involved and the extent of fastness achievable is worth investigating.

- The error correcting capability of the codes obtained by using the entended CRJT for finite fields is an important direction to pursue.

\section{REFERENCES}

[1] N. Ahmed and K. R. Rao, Orthogonal Transforms for Digital Signal Processing. New York: Springer-Verlag, 1975.

[2] W. K. Pratt, J. Kane, and H. C. Andrews, "Hadamard transform image coding," Proc. IEEE, vol. 57, pp. 58-68, 1969.

[3] K. G. Beauchamp, Walsh Functions and Their Applications. New York: Academic, 1975.

[4] F. J. MacWilliams and N. J. A. Sloane, The Theory of Error-Correcting Codes. Amsterdam, The Netherlands: North-Holland, 1988.

[5] M. H. Lee, "The center weighted Hadamard transform," IEEE Trans. Circuits Syst., vol. CAS-36, pp. 1247-1249, Sept. 1989.

[6] - "Fast complex reverse jacket transform," in Proc. 22nd Symp. Information Theory and Its Application (SITA99), Yuzawa, Niigata, Japan, Nov. 30-Dec. 31999.

[7] - "A new reverse jacket transform and its fast algorithm," IEEE Trans. Circuits Syst. II, vol. 47, pp. 39-46, Jan. 2000.

[8] M. H. Lee, J. Y. Park, and S. Y. Hong, "A simple binary index generation for reverse jacket sequence," in Proc. Int. Symp. Information Theory and Applications (ISITA), Hawaii, Nov. 5-9, 2000, pp. 429-433.

[9] M. H. Lee, "A new reverse jacket transform based on Hadamard matrix," in Proc. Int. Symp. Information Theory, Sorrento, Italy, June 25-30, 2000, p. 471

[10] B. S. Rajan and M. H. Lee, "Quasicyclic dyadic codes in Walsh-Hadamard transform domain," IEEE Trans. Inform. Theory, submitted for publication.

[11] K. R. Rao and J. J. Hwang, Techniques and Standards for Image Video and Audio Coding. Englewood Cliffs, NJ: Prentice-Hall, 1996.

[12] R. J. Clark, Transform Coding of Images. New York: Academic, 1985.

[13] R. E. Blahut, Theory and Practice of Error Control Codes. Reading, MA: Addison-Wesley, 1982. 
[14] M. G. Parker and M. H. Lee, "Optimal bipolar sequences for the complex reverse jacket transform," in Proc. Int. Symp. Information Theory Applications, (ISITA), vol. 1, Hawaii, Nov. 5-10, 2000, pp. 425-428.

[15] M. H. Lee, "Revisited DFT matrix via the reverse jacket transform and its application to communications," in Proc. 22nd Symp. Information Theory Its Applications (SITA99), Yuzawa, Niigata, Japan, Nov. 30-Dec. 3, 1999.

[16] S. Mister and C. Adams, "Practical S-box design," in Workshop Record Selected Areas Cryptography (SAC'96), Aug. 15-16, 1996, pp. 61-76.

[17] R. K. Yarlagadda and J. E. Hershey, Hadamard Matrix Analysis and Synthesis With Applications to Communications and Signal/Image Processing. Boston, MA: Kluwer, 1997.

[18] A. K. Jain, Fundamentals of Digital Image Processing. Englewood Cliffs, NJ: Prentice-Hall, 1989.

[19] M. H. Lee, "Fast complex reverse jacket transform," IEEE Trans. Circuits Syst. II, submitted for publication.

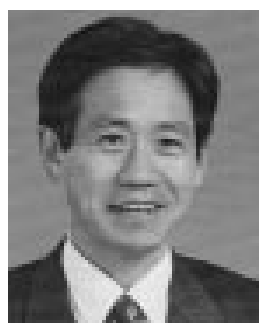

Moon Ho Lee (S'81-M'85-SM'86) received the B.S. and M.S. degrees in electrical engineering from the Chonbuk National University, Korea, in 1967 and 1976, respectively, and the Ph.D. degrees in electronics engineering from the Chonnam National University in 1984 and the University of Tokyo, Japan, in 1990.

From 1970 to 1980 , he was a chief engineer with Namyang Moonhwa Broadcasting. Since 1980, he has been a professor with the Department of Information and Communication and a Director with the Institute of Information and Communication, both at Chonbuk National University. From 1985 to 1986, he was also with the University of Minnesota, as a Postdoctoral Fellow. He has held visiting positions with the University of Hannover, Germany, during 1990, the University of Aachen, Germany, during 1992 and 1996, and the University of Munich, Germany, during 1998. He has authored 13 books, including Digital Communications (Korea: Youngil, 1999), Information and Coding (Dorea: Bokdu, 1998), Digital Image Processing, (Korea: Daeyoung, 1994), and Digital Filter Design (Korea: Daeyoung, 1995). His research interests include multidimensional source and channel coding, mobile communication; and image processing.

Dr. Lee is a Registered Telecommunication Professional Engineer and a member of the National Academy of Engineering in Korea. He was the recipient of the paper prize award from the Korean Institute of Communication Science in 1986 and 1987, the Korean Institute of Electronics Engineers in 1987, and Chonbuk Province in 1992.

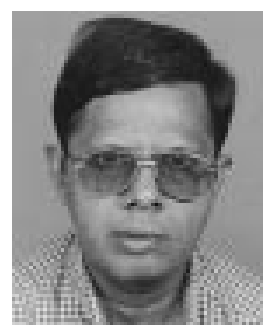

B. Sundar Rajan (S'84-M'91-SM'98) was born in India. He received the B.Sc. degree in mathematics from Madras University, India, the B.Tech. degree in electronics from Madras Institute of Technology, and the M.Tech. and Ph.D. degrees in electrical engineering from the Indian Institute of Technology, Kanpur, India, in 1979, 1982, 1984, and 1989, respectively.

He was a faculty member with the Department of Electrical Engineering at the Indian Institute of Technology, Delhi, India, from 1990 to 1997. Currently, he is an associate professor with the Department of Electrical Communication Engineering and the Department of Computer Science and Automation, Indian Institute of Science, Bangalore, India. His primary research interests are in error-control coding, coded modulation, and cryptology.

Dr. Rajan is a member of the American Mathematical Society.

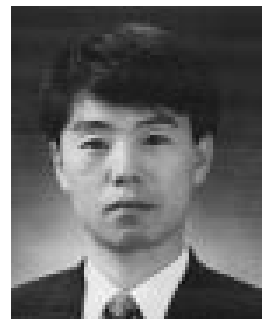

Ju Yong Park (M’01) was born in Jeonju, Korea, in 1958. He received the B.S., M.S., and Ph.D. degrees in electronics engineering from Chonbuk National University, Chonbuk, Korea, in 1982, 1986, and 1994, respectively.

Since 1991, he has been an associate professor with the Department of Electronics and Multimedia Engineering at Seonam University, Chonbuk, Korea. His current research interests include wireless communications, WCDMA, coding, and ATM networks. 